\title{
Diversifying the Ocean Sciences THOUGHTS ON THE CHALLENGE AHEAD
}

\author{
By Corey Garza
}

The lack of diversity in the ocean sciences is not a particularly big secret or a recent issue. In his inaugural column in 2000 for The Oceanography Classroom, Dean McManus wrote: "A particular challenge for higher education is to include more members of underrepresented groups in the study of the ocean. Fifteen years from now, $40 \%$ of the traditional undergraduate-age population will consist of these underrepresented groups, but today the ocean sciences have the lowest participation by underrepresented groups of any science." Despite this prescient statement from McManus, little progress has been made in diversifying the ranks of the ocean sciences. In a recent National Science Foundation survey of current graduate students in the ocean sciences, approximately $8.8 \%$ of those surveyed identify as coming from a group that is considered part of a minority community in the United States (Black, Hispanic/Latinx, Native Alaskan, Native American, Pacific Islander). As might be expected, these numbers are even worse for the professional ranks of agencies, nonprofits, and university programs that focus on the ocean sciences. The lack of these communities' representation in ocean science careers is underscored by the past year's news reports, brimming as they have been with the brutality and injustices that those in BIPOC (Black, Indigenous, People of Color) communities face daily. The STEM community, including the ocean sciences, is now, more than ever, being forced to confront the ways in which its institutions and culture contribute to the inequities facing BIPOC communities. Here, I draw upon my dual identity as a member of the ocean science and Latinx communities to discuss how the ocean sciences can enact meaningful changes around improving diversity, equity, and inclusion (DEI).

Over the past 18 years of my career, I have had the privilege of leading numerous efforts focused on improving DEI in ocean science. Having benefited from STEM-based DEI programs as a student, I feel a unique obligation to pay these efforts forward. I engage in these efforts to provide opportunities for those who have historically been absent from the ocean sciences and to train a new generation of scientists who bring diverse perspectives to solving the most pressing issues facing twenty-first century ocean science. My time working on issues surrounding the lack of diversity in the ocean sciences has helped me identify a few obstacles faced by individuals who may wish to enter the discipline. These include, but are not limited to, (1) messaging about career options and opportunities in the ocean sciences that may not resonate with BIPOC populations, (2) depictions of how research in ocean science is conducted, (3) lack of engagement of BIPOC scientists in the research enterprise, and (4) the reward structure of ocean science.

One of the biggest issues facing the ocean sciences centers around how we craft messaging about what the field entails and what career options exist. Relative to other fields, such as the biomedical sciences, the ocean sciences have struggled with messaging and strategies for engaging students early in their academic careers. One simply needs to look at depictions of medical scientists in television and film, with many being portrayed as having successful careers that benefit large segments of society. Such depictions of ocean scientists in the media are rare, with the most common being that of the explorer venturing out to sea with a largely undefined purpose. If you were to ask a room full of ocean scientists what an ocean scientist does, you are likely to get a multitude of answers, ranging from oceanography to engineering to biology. Thus, as a field, we lack the type of clear messaging about our discipline that other fields successfully employ to engage with underrepresented students. Coupled with our messaging issue is the tendency to direct students to academic careers. There is ample evidence that suggests students in BIPOC communities may elect careers such as medicine not only because of the clear career options but also because these careers can be seen as ways for students to help their own communities. Beyond improving how we portray the ocean sciences to non-STEM-oriented communities, the ocean sciences must improve how we depict career paths in our disciplines to better engage with the diverse culture value structures of BIPOC students.

Another challenge the ocean sciences face in attracting diverse individuals is due in part to how research is depicted within the disciplines. My own interest in ocean science was driven in part 
by images of ocean scientists donning scuba gear or getting into submersibles and venturing down into the unexplored. While these portrayals can be attractive to broad swath of students, they can also act as barriers to inclusion that our community may not have previously considered. I well remember the costs of purchasing my first set of scuba gear and paying for my introductory scuba course. Such a financial burden can be a significant barrier for low-income students who may not have the resources to invest in this type of purchase. Additionally, ocean scientists are overwhelming presented as able bodied, suggesting that those with limited mobility cannot engage in ocean science research. Our community needs to reimagine how we present our field to prospective students and develop more inclusive representations that consider the financial and physical barriers that may exclude whole groups of students.

Beyond focusing on the inclusion of diverse students, our field must also examine how the research culture of the ocean sciences may exclude those who identify as BIPOC and how our reward structures value DEI activities. For instance, Top Tier Research Institutions $(\mathrm{R} 1)^{1}$ have historically received the bulk of research funding from agencies such as the National Institutes of Health and the National Science Foundation, creating an imbalance in the funding portfolios for the agencies that support basic research in the United States. When minorityserving institutions (MSIs) are included in proposals with $\mathrm{R} 1$ institutions, their participation is most often defined as broader-impact partners whose primary roles are to conduct outreach activities associated with a research grant. The combination of these factors results in
MSIs and their faculties, who may often come from minority groups, receiving little research support through the major funding agencies. Major research institutions must develop new strategies for engaging with MSIs beyond regarding them as broader-impact partners. MSIs not only serve underrepresented student populations, they also host high caliber research faculties and programsthe NOAA Cooperative Science Centers, for example, represent one pathway through which R1 institutions can begin to reframe their interactions with MSIs.

In addition to how few minority research scientists are available as role models, the reward structure in ocean science can also lead to the lack of inclusivity in the discipline. For example, reward structures in academia tend to value basic research over solutions and applied research, while students from minority communities may be more drawn to careers and research interests that present opportunities to contribute to the public good. Higher education research demonstrates that when research institutions, their leadership, and funding agencies revisit definitions of excellence that align with equity and diversity, momentum is created for change throughout the system. A focus on research that is not purely discovery-based science is often at odds with the reward structures inherent in universities, journals, and funding agencies. Scientists from underrepresented groups may prefer to work in other sectors that are more in line with their cultural value systems, thus further contributing to the lack of diversity in ocean science.

While the items discussed in this column are not exhaustive or a cure-all for the lack of diversity in ocean science, my hope is they spark discussions around ways that we can begin to improve representation in our field. They should not be examined in isolation but in concert with each other as part of larger coordinated efforts to improve representation in the ocean sciences. This is a crucial time to broaden participation and to raise the profile of ocean science among underrepresented populations. Modern human activities have resulted in a widely accepted new geological epoch, the Anthropocene, that directly impacts the ocean. Many of the global phenomena associated with these changes will have deleterious impacts on society and will require the participation of diverse individuals to help find novel solutions to counteract them. A homogeneous community of ocean scientists that lacks representation of diverse groups limits the likelihood that the urgent challenges associated with the Anthropocene will be met, while also decreasing the likelihood that findings will be accepted by an increasingly diverse public. Now is the time for the ocean sciences to engage a new generation of diverse individuals who can bring new perspectives and approaches for dealing with the emerging needs of twenty-first century ocean science. [

\section{AUTHOR}

Corey Garza (cogarza@csumb.edu) is Professor, Department of Marine Science, and Director, Coastal and Maine Ecosystems Programs, California State University, Monterey Bay, Seaside, CA, USA.

\section{ARTICLE DOI}

https://doi.org/10.5670/oceanog.2021.204

\footnotetext{
1 "Top tier" or "R1" universities are doctoral degree granting institutions with "very high research activity" according to the Carnegie Classification of Institutions of Higher Education, the main framework for recognizing and describing institutional diversity in US higher education since 1970, when the Carnegie Commission on Higher Education developed a classification of colleges and universities to support its program of research and policy analysis.
} 